\title{
Awareness that Dogs Can Be Carriers for Ticks that Transmit Lyme Disease
}

\author{
Joshua Fogel and Sherilyne Co \\ Department of Business Management, Brooklyn College
}

\begin{abstract}
Background and Purpose: Tick exposure places one at possible risk for Lyme disease. Awareness of this fact can possibly prevent its occurrence. Urban college students are often in outdoor areas where there is potential tick exposure. Methods: College students in New York City $(n=714)$ were surveyed about demographics, Lyme-disease knowledge, Lyme disease related topics, and dog-specific items. Multivariate logistic regression analyses were conducted to identify factors that are related to awareness that dogs can be carriers for ticks that transmit Lyme disease. Results: For both dog owners and those who do not own dogs, certain Lyme disease knowledge topics, previously heard of Lyme disease, and aware of a Lyme disease dog vaccine were each associated with increased odds for awareness about dogs being carriers. Among dog owners, African American race/ethnicity and knowing someone diagnosed with Lyme disease were each associated with increased odds for awareness, while previous diagnosis of Lyme disease had decreased odds for awareness. Conclusion: One-on-one education by physicians, nurses, veterinarians, healthcare practitioners and public health practitioners could help address some of the lack of awareness among urban college students that dogs can be carriers for ticks that can transmit Lyme disease.
\end{abstract}

(c) 2016 Californian Journal of Health Promotion. All rights reserved. Keywords: vaccines, dogs, Lyme disease, ticks, awareness, knowledge

\section{Introduction}

Lyme disease is the most common vector-borne illness in the United States (Centers for Disease Control and Prevention (CDC), 2015). Lyme disease is transmitted to humans when humans are bitten by ticks that are infected with the bacteria for Lyme disease (Hofhuis, Harms, Bennema, van den Wijngaard \& van Pelt, 2015). There were an estimated 329,000 Lyme disease cases each year in the United States from 20052010 (Nelson, Saha, Kugeler, Delorey, Shankar, Hinckley \& Mead, 2015). In 2013, 95\% of the human Lyme disease cases in the United States were from states in the northeast and upper Midwest regions (CDC, 2015).

A number of studies have examined knowledge about Lyme disease. College students from Michigan and West Virginia, on average, scored correct answers for two-thirds of the questions about knowledge of Lyme disease. Women had greater knowledge than men (Plackowski, Hull, \& Hull, 2014). More than one third of participants in a study in the Netherlands correctly answered 4 out of 5 Lyme disease knowledge statements (Beaujean, Bults, van Steenbergen \& Voeten, 2013). In a crosscultural study, almost two thirds of Swiss participants knew that Lyme disease is transmitted by a tick while less than one third of Canadian participants were knowledgeable about this topic. In a Lyme disease study, Swiss women ages 35-54 years had greater knowledge than their male counterparts, but this did not occur among the Canadian participants (Aenishaenslin, Ravel, Michel, Gern, Milord, Waaub et al., 2014). In a study conducted in Martha's Vineyard, Massachusetts, almost half correctly answered Lyme disease knowledge questions while one tenth chose the answer of "did not know" for all 9 Lyme disease knowledge questions (Valente, Wemple, Ramos, Cashman \& Savageau, 2015).

Canine seroprevalence is strongly correlated to human Lyme disease incidence (Mead, Goel, Kugeler, 2011; Little, Beall, Bowman, 
Chandrashekar \& Stamaris, 2014). Pets such as dogs do not directly transmit Lyme disease to humans. However, pets can carry ticks infected with Lyme disease into the yard or home (CDC, 2014). In a study conducted in Ithaca, New York, among 100 dog owners of which 85\% were age 25 or below, one tenth incorrectly reported that dogs can directly transmit Lyme disease to humans (Sandhu \& Singh, 2015). Owning dogs and/or cats was associated with increased odds to engage in Lyme disease preventive behaviors in low-incidence Lyme disease states but not in high-incidence Lyme disease states (Herrington, 2004).

There is limited research on the topic of correct knowledge for dogs and transmission of Lyme disease to humans. Furthermore, we are not aware of any research on awareness that dogs can be carriers for ticks that can transmit Lyme disease. Studying such topics is important and can contribute to public health as correct knowledge and awareness about Lyme disease topics can relate to use of proper Lyme disease preventive behaviors. Lower levels of correct knowledge and awareness about Lyme disease topics would suggest the need for public health promotion programs to disseminate correct knowledge. This study has two aims. One study aim is to understand undergraduate students' knowledge about Lyme disease topics. The second study aim is to stratify analyses by dog ownership (those who own dogs and those who do not) to determine if predictors of awareness that dogs can be carriers for ticks that can transmit Lyme disease vary by this variable.

\section{Methods}

\section{Participants and Procedures}

There were 757 people approached to partake in the anonymous survey which was administered in a public college in New York City. There were 21 people who declined to participate. From the 736 surveys completed, there was a $97.2 \%$ response rate. We did not include 22 individuals who were above the age of 36 to maintain a more conventional college-aged group. We analyzed data from 714 participants. This cross-sectional study used a convenience sample. Ethical approval to conduct this study was received from the college Institutional Review Board Human Research Protections Program. Oral informed consent was obtained from all participants. With permission from the instructor, the surveys were distributed and completed anonymously in classrooms. The survey took approximately 5 minutes to complete. Data collection occurred from September through October 2014.

\section{Measures \\ Demographics}

The items measured were age (years), sex (man, woman), and race/ethnicity (White, African American, Hispanic, Asian, South Asian or Other).

\section{Lyme Disease Knowledge}

Lyme disease knowledge consisted of five separate true or false questions. Four questions were obtained from an earlier article (Beaujean et al., 2013). The reverse code of false as the correct answer was used for the knowledge question, "Ticks mostly fall out of trees." The other knowledge questions with correct answers of true are, "People can get Lyme disease after a tick bite, "The chance for tick bites is higher during the summer than the winter," and "Using insect repellent on skin can protect against tick bites." In addition, we created the question of, "The insect that causes Lyme disease is typically as large as an adult common housefly," with the correct answer of true based upon available information from the CDC website (CDC, 2014).

\section{Lyme Disease Related Topics}

There were five yes/no items that were created to assess topics that are related to the transmission of Lyme disease. They were the following: "I typically spend a lot of time outdoors," "Before participating in this survey, have you ever heard of Lyme disease?," "Have you ever been diagnosed with Lyme disease?,” "Do you know someone who was diagnosed with Lyme disease?," and "Have you ever had a tick bite?”

\section{Dog-specific Items}

There were two dog-specific separate yes/no items. These were, "Do you own a dog?” and 
"Are you aware that there is a vaccine for dog (canine) Lyme disease?”

\section{Outcome Variable}

The outcome variable was the yes/no item of, "Are you aware that dogs can be carriers for ticks that transmit Lyme disease?”

\section{Statistical Analyses}

The mean and standard deviation was calculated for participants' age, while frequencies and percentages were calculated for the categorical variables. The outcome of awareness that dogs can be carriers for the tick that transmits Lyme disease was analyzed with multivariate logistic regression. The predictors consisted of demographics, Lyme-disease knowledge, Lyme disease related topics, and dog-specific variables. These analyses were repeated separately for both dog owners and those who did not own dogs. Also, a Pearson chi-square statistic was calculated to compare dog owners to those who did not own dogs for awareness that dogs can be carriers for the tick that transmits Lyme disease. All p-values were twotailed. STATA SE version 13 was used for all analyses (StataCorp LP, 2013).

\section{Results}

Table 1 describes the overall sample characteristics. Mean age was slightly above 22 years ( $\mathrm{SD}=3.53$ years). There were an equal number of men and women $(n=357$ for each gender). For race/ethnicity, more than one-third were white (35.6\%, $\mathrm{n}=254)$, more than onequarter were Asian (25.9\%, $\mathrm{n}=185$ ) and more than one-third were either African American, Hispanic, South Asian or Other. For the two knowledge items of people can get Lyme disease after a tick bite $(82.9 \%, n=592)$ and the chance for tick bites is higher during the summer than the winter ( $80.5 \%, n=575)$, more than four-fifths correctly responded true. Other knowledge items had lower rates of correct responding. For example, less than two thirds correctly responded false to the question that ticks mostly fall out of trees $(60.2 \%, n=430)$. Slightly more than one half correctly responded true to the question that using insect repellent on skin can protect against tick bites $(52.8 \%, \mathrm{n}=377)$. Less than one half correctly responded true that ticks are as large as an adult common housefly $(41.3 \%, n=295)$.

Table 1

Characteristics of the Sample of 714 Dog Owners and NonDog Owners

\begin{tabular}{lr}
\hline \multicolumn{2}{c}{ Variable } \\
\hline Sex (women) & Demographics $(\%)$ \\
Race/ethnicity & $357(50.0 \%)$ \\
White & $254(35.6 \%)$ \\
African American & $102(14.3 \%)$ \\
Hispanic & $66(9.2 \%)$ \\
Asian & $185(25.9 \%)$ \\
South Asian & $46(6.4 \%)$ \\
Other & $60(8.4 \%)$ \\
Missing & $1(0.1 \%)$
\end{tabular}

Knowledge (correct)

Can get Lyme disease after a tick bite

$592(82.9 \%)$

Tick bites higher during summer than winter

Ticks mostly fall out of trees (reverse)

$575(80.5 \%)$

Insect repellent protects against tick bites

$430(60.2 \%)$

Insect that causes Lyme disease is large as an adult common housefly

$295(41.3 \%)$

Lyme disease related topics (yes)

Typically spend a lot of time outdoors

$400(56.0 \%)$

Before survey, ever heard of Lyme disease

Ever diagnosed with Lyme disease

Know someone diagnosed with Lyme disease

Ever had tick bite

$448(62.7 \%)$

$12(1.7 \%)$

$104(14.6 \%)$

$88(12.3 \%)$

Own a dog

Dog-specific (yes)

Aware of dog (canine) Lyme disease vaccine

$155(21.7 \%)$

$205(28.7 \%)$

Outcome variable

Are you aware that dogs can be carriers for ticks that transmit Lyme disease? (yes)

$377(52.8 \%)$

Note: Sample sizes slightly vary due to missing data.

With regard to the Lyme disease related topics, more than one half typically spend a lot of time outdoors $(56.0 \%, \mathrm{n}=400)$ and less than two thirds heard of Lyme disease before participating in the survey $(62.7 \%, n=448)$. Less than $2 \%$ were ever diagnosed with Lyme disease $(1.7 \%, \mathrm{n}=12)$. Less than one fifth knew someone who was diagnosed with Lyme disease (14.6\%, $\mathrm{n}=104)$ whereas slightly more than one tenth had a tick bite $(12.3 \%, n=88)$. With regard to the dog items, slightly more than one fifth owned a dog $(21.7 \%, n=155)$ and more than one quarter were aware that there is a vaccine for dog Lyme 
disease $(28.7 \%, \mathrm{n}=205)$. Also, more than one half $(52.8 \%, n=377)$ were aware that dogs can be carriers for ticks that transmit Lyme disease.

Pearson chi-square analysis comparing dog owners and those who did not own dogs had a statistically significant percentage difference $(\mathrm{p}<0.001) \quad$ where dog owners $(67.1 \%$, $n=104 / 155$ ) had greater awareness that dogs can be carriers for ticks that transmit Lyme disease than those who did not own dogs (48.8\%, $n=273 / 559)$.

Table 2

\begin{tabular}{|c|c|c|}
\hline Variable & $\begin{array}{l}\text { Dog owner } \\
\text { OR (95\% CI) }\end{array}$ & $\begin{array}{l}\text { Non-dog owner } \\
\text { OR (95\% CI) }\end{array}$ \\
\hline Demographics & & \\
\hline Age (years) & $0.88(0.76,1.02)$ & $0.97(0.92,1.03)$ \\
\hline \multicolumn{3}{|l|}{ Sex } \\
\hline Men & 1.00 & 1.00 \\
\hline Women & $1.45(0.49,4.33)$ & $1.01(0.68,1.51)$ \\
\hline \multicolumn{3}{|l|}{ Race/ethnicity } \\
\hline White & 1.00 & 1.00 \\
\hline African American & $17.89(3.22,99.44)^{* *}$ & $1.41(0.74,2.68)$ \\
\hline Hispanic & $1.64(0.32,8.32)$ & $0.91(0.43,1.93)$ \\
\hline Asian & $2.23(0.54,9.25)$ & $0.83(0.50,1.38)$ \\
\hline South Asian & $0.97(0.04,25.59)$ & $0.71(0.32,1.56)$ \\
\hline Other & $16.29(1.29,206.01)^{*}$ & $1.15(0.54,2.49)$ \\
\hline \multicolumn{3}{|l|}{ Knowledge (correct) } \\
\hline Can get Lyme disease after a tick bite & $6.16(1.34,28.35)^{*}$ & $3.34(1.85,6.05)^{* * *}$ \\
\hline Tick bites higher during summer than winter & $0.46(0.10,2.04)$ & $1.51(0.90,2.53)$ \\
\hline Ticks mostly fall out of trees (reverse) & $5.77(1.81,18.47)^{* *}$ & $1.55(1.03,2.34)^{*}$ \\
\hline Insect repellent protects against tick bites & $3.12(1.06,9.23)^{*}$ & $0.70(0.47,1.04)$ \\
\hline \multicolumn{3}{|l|}{ Insect that causes Lyme disease is large as an adult common } \\
\hline housefly & $1.45(0.50,4.16)$ & $1.16(0.77,1.76)$ \\
\hline \multicolumn{3}{|l|}{ Lyme disease related topics (yes) } \\
\hline Typically spend a lot of time outdoors & $1.32(0.42,4.13)$ & $0.99(0.66,1.47)$ \\
\hline Before survey, ever heard of Lyme disease & $3.65(1.20,11.10)^{*}$ & $1.88(1.22,2.88)^{* *}$ \\
\hline Ever diagnosed with Lyme disease & $0.06(0.01,0.55)^{*}$ & N/A \\
\hline Know someone diagnosed with Lyme disease & $5.50(1.21,25.05)^{*}$ & $1.31(0.70,2.45)$ \\
\hline Ever had tick bite & $1.56(0.43,5.64)$ & $1.75(0.88,3.46)$ \\
\hline \multicolumn{3}{|l|}{ Dog-specific (yes) } \\
\hline Aware of dog (canine) Lyme disease vaccine & $15.58(4.97,48.84)^{* * *}$ & $12.23(6.25,23.95) * * *$ \\
\hline
\end{tabular}

Note: $\mathrm{OR}=$ odds ratio, $\mathrm{CI}=$ confidence interval, N/A=not applicable. For non-dog owner, the question of, "ever diagnosed with Lyme disease" was excluded by the statistical analysis package from the analysis since it predicted successes perfectly.

Pseudo R square: Dog owner $=0.40$, Non-dog owner $=0.20$.

${ }^{*} \mathrm{p}<0.05,{ }^{* *} \mathrm{p}<0.01,{ }^{* * *} \mathrm{p}<0.001$

Table 2 shows the multivariate logistic regression analyses for predictors for awareness that dogs can be carriers for ticks that transmit Lyme disease. Due to listwise deletion the multivariate analysis had 3 fewer participants for dog owners and 16 fewer participants for nondog owners. No imputation was done. For dog owners, African American and Other race/ethnicity, correct responses to the knowledge items of one can get Lyme disease after a tick bite, that ticks mostly fall out of trees, and using insect repellant on skin can protect against tick bites, the Lyme disease topic of having heard of Lyme disease before the survey, and awareness that there is a vaccine for dog Lyme disease all had statistically significant increased odds for awareness. Although knowing someone diagnosed with Lyme disease had statistically significant increased odds for awareness, ever been diagnosed with Lyme disease had statistically significant decreased odds for awareness. This pattern of associations was similar among non-dog owners, with the exception of no significant associations of African American and Other race/ethnicity, 
correct response to the knowledge item of using insect repellant on skin can protect against tick bites, and knowing someone diagnosed with Lyme disease with awareness. Also, for non-dog owners, ever diagnosed with Lyme disease was excluded from the analysis by the statistical analysis package due to predicting successes perfectly.

\section{Discussion}

The purpose of the study was to understand knowledge about Lyme disease topics and variables associated with awareness that dogs can be carriers for ticks that can transmit Lyme disease. Correct knowledge and awareness about Lyme disease topics are important as this can relate to use of proper Lyme disease preventive behaviors.

For demographics, we found that African American and Other race/ethnicity dog owners had increased odds for awareness that dogs can be carriers for ticks that transmit Lyme disease. No association was found for Hispanic, Asian, or South Asian dog owners and also for those from any race/ethnicity who did not own dogs. We are not aware of any literature on increased knowledge for Lyme disease among African Americans. African Americans have lower incidence of Lyme disease than whites (Fix, Peña, \& Strickland, 2000). With regard to pets, African American pregnant women have less dog ownership than their White counterparts (Ezell, Cassidy-Bushrow, Havstad et al., 2014), and also African American veterinary students have lower pet attachment than Whites (Brown, 2002). These data from Lyme disease incidence and pet ownership suggest that Whites would be more knowledgeable about dog-related Lyme disease topics due to greater personal relevance. Also, there is extensive literature that African Americans have greater health disparities than Whites for many diseases (Smedley, Stith, \& Nelson, 2002; Taneja, So, Stewart, Evans, \& Jason, 2015). Apparently African Americans have higher knowledge than Whites about Lyme disease transmission as it relates to dogs. From an intervention perspective, public health educational programs about awareness that dogs can be carriers for ticks that transmit Lyme disease could benefit Hispanic, Asian, or South Asian dog owners and also those from all racial/ethnic groups who do not own dogs.

We found that correct knowledge ranged from $41.3 \%$ to $82.9 \%$ across the various topics. The association between correct knowledge that people can get Lyme disease after a tick bite and that ticks mostly do not fall out of trees with increased awareness that dogs can be carriers for ticks that transmit Lyme disease was present for both dog owners and non-dog owners. We are not aware of any previous studies on this topic. Dogs can transmit rabies to humans. In a sample of dog owners and those who did not own dogs, correct knowledge for an item related to rabies was associated with proper rabies preventive behaviors related to dogs transmitting rabies to humans (Bingham, Budke, \& Slater, 2010). We add to the literature and report that correct knowledge for Lyme disease is associated with increased awareness that dogs can be carriers for ticks that transmit Lyme disease to humans. From an intervention perspective, public health educational programs could be useful to disseminate accurate information about awareness that dogs can be carriers for ticks that transmit Lyme disease. This could result in higher levels of correct knowledge.

We found that for both dog owners and non-dog owners, having heard about Lyme disease before the survey and also being aware that there is a vaccine for dog Lyme disease were each associated with increased odds for awareness that dogs can be carriers for ticks that transmit Lyme disease. We are not aware of any previous literature on this topic with regard to Lyme disease. In a different study, those who heard about cardiovascular diseases had more accurate knowledge about certain risk factors for cardiovascular disease (Tedesco, di Giuseppe Napolitano \& Angelillo, 2015). We add to this literature and show that having heard about Lyme disease or being aware of a vaccine for dog Lyme disease is associated with increased awareness that dogs can be carriers for ticks that transmit Lyme disease to humans. From an intervention perspective, this demonstrates the public health importance of teaching information about both Lyme disease and the vaccine for dog 
Lyme disease, as this can result in greater awareness that dogs can be carriers for ticks that transmit Lyme disease.

There were two findings for Lyme diseaserelated topics where dog owners had a different pattern that those who did not own dogs for the outcome of interest. We found that dog owners who knew someone previously diagnosed with Lyme disease had increased odds for awareness that dogs can be carriers for ticks that transmit Lyme disease. The opposite pattern occurred for personal history of Lyme disease, where those who had ever been diagnosed with Lyme disease had decreased odds for awareness that dogs can be carriers for ticks that transmit Lyme disease. These associations were not found for those who did not own dogs. Possible reasons for understanding the finding for ever been diagnosed with Lyme disease and decreased awareness among dog owners is that there is either a possible pattern of helplessness to prevent Lyme disease reoccurrence or a sense of protective immunity for Lyme disease reoccurrence.

\section{Limitations}

This study has some limitations worth noting. First, our sample consisted of college students, which restricted the age range of our sample. Future research should include a sample with varied ages to determine if these findings are consistent with other age groups. Second, although we asked whether one typically spends a lot of time outdoors, we did not ask about participation in particular outdoor activities, which may be associated with better awareness.
Third, although we asked about dog ownership, we did not ask about contact with dogs despite not owning a dog. Regular contact with a dog may influence the amount of knowledge about dog-related diseases. Fourth, self-reported knowledge of awareness that dogs can be carriers for ticks that carry Lyme disease could be biased where some participants may have responded yes even though they truly were not aware.

\section{Conclusion}

In conclusion, we found that general knowledge about Lyme disease was associated with increased awareness that dogs can be carriers for ticks that transmit Lyme disease. Dog owners who were previously diagnosed with Lyme disease had decreased odds for awareness that dogs can be carriers. Those who heard of Lyme disease before the survey and those who were aware of a dog vaccine for Lyme disease had greater odds for awareness that dogs can be carriers for ticks that can transmit Lyme disease. Physicians, nurses, veterinarians, healthcare practitioners and public health practitioners should consider direct one-on-one education about Lyme disease, particularly for those who own dogs and especially those who own dogs and who were previously diagnosed with Lyme disease. Also, education should be provided about the available tick control approaches for dogs. Public health campaigns about Lyme disease targeting dog owners could help address some of the lack of awareness that dogs can be carriers for ticks that can transmit Lyme disease.

\section{References}

Aenishaenslin, C., Ravel, A., Michel, P., Gern, L., Milord, F., Waaub, J.-P., \& Bélanger, D. (2014). From Lyme disease emergence to endemicity: A cross sectional comparative study of risk perceptions in different populations. BMC Public Health, 14, 1298.

Beaujean, D. J. M. A., Bults, M., van Steenbergen, J. E., \& Voeten, H. A. C. M. (2013). Study on public perceptions and protective behaviors regarding Lyme disease among the general public in the Netherlands: Implications for prevention programs. BMC Public Health, 13, 225.

Bingham, G. M., Budke, C. M. \& Slater, M. R. (2010). Knowledge and perceptions of dog-associated zoonoses: Brazos County, Texas, USA. Preventive Veterinary Medicine, 93, 211-221.

Brown, S-E. (2002). Ethnic variations in pet attachment among students at an American school of veterinary medicine. Society and Animals, 10(3), 249-266.

Centers for Disease Control and Prevention (2014). Lyme disease: Transmission. Available at: http://www.cdc.gov/lyme/transmission/index.html . Accessed November 4, 2014. 
Centers for Disease Control and Prevention (2015). Lyme disease: Data and statistics. Available at: http://www.cdc.gov/lyme/stats/index.html . Accessed September 17, 2015.

Ezell, J. M., Cassidy-Bushrow, A. E., Havstad, S., Joseph, C. L. M., Wegienka, G., Jones, K., Ownby, D. R. \& Johnson, C. C. (2014). Prenatal dog-keeping practices vary by race: Speculations on implications for disparities in childhood health and disease. Ethnicity and Disease, 24, 104-109.

Fix, A. D., Peña, C. A. \& Strickland, G. T. (2000). Racial differences in reported Lyme disease incidence. American Journal of Epidemiology, 152(8), 756-759.

Herrington, J. E., Jr. (2004). Risk perceptions regarding ticks and Lyme disease: A national survey. American Journal of Preventive Medicine, 26(2), 135-140.

Hofhuis, A., Harms, M., Bennema, S., van den Wijngaard, C. C., \& van Pelt, W. (2015). Physician reported incidence of early and late Lyme borreliosis. Parasites and Vectors, 8, 161.

Little, S. E., Beall, M. J., Bowman, D. D., Chandrashekar, R., \& Stamaris, J. (2014). Canine infection with Dirofilaria immitis, Borrelia burgdorferi, Anaplasma spp., and Ehrlichia spp. in the United States, 2010- 2012. Parasite and Vectors, 7, 257.

Mead, P., Goel, R., \& Kugeler, K. (2011). Canine serology as adjunct to human Lyme disease surveillance. Emerging Infectious Diseases, 17(9), 1710- 1712.

Nelson, C. A., Saha, S., Kugeler, K. J., Delorey, M. J., Shankar, M. B., Hinkley, A. F., \& Mead, P. S. (2015). Incidence of clinician-diagnosed Lyme disease, United States, 2005-2010. Emerging Infectious Diseases, 21(9), 1625-1631.

Plackowski, E., Hull, D., \& Hull, J. (2014). Knowledge, attitudes, and behavioral intentions toward those with Lyme disease. International Journal of Education and Social Science, 1(4), 26-30.

Sandhu, G. K. \& Singh, D. (2014). Level of awareness regarding some zoonotic diseases, among dog owners of Ithaca, New York. Journal of Family Medicine and Primary Care, 3(4), 418- 423.

Smedley, B.D., Stith, A.Y. \& Nelson, A. R. (Eds.). (2003). Unequal treatment: Confronting racial and ethnic disparities in health care. Washington, D.C.: The National Academies Press.

StataCorp (2013). Stata Statistical Software: Release 13. College Station, TX: StataCorp LP.

Taneja, I., So, S., Stewart, J. M., Evans, M., \& Jason, L. A. (2015). Prevalence and severity of symptoms in a sample of African Americans and White participants. Journal of Cultural Diversity, 22(2), 50-58.

Tedesco, L. M. R., di Giuseppe, G., Napolitano, F. \& Angelillo, I. F. (2015). Cardiovascular diseases and women: Knowledge, attitudes, and behavior in the general population in Italy. BioMed Research International, 2015, Article ID 324692.

Valente, S. L., Wemple, D., Ramos, S., Cashman, S. B., \& Savageau, J. A. (2015). Preventive behaviors and knowledge of tick-borne illnesses: Results of a survey from an endemic area. Journal of Public Health Management and Practice, 21(3), E16-E23.

\author{
Author Information \\ * Dr. Joshua Fogel \\ Brooklyn College of the City University of New York \\ Department of Business Management, 218A \\ 2900 Bedford Avenue \\ Brooklyn, NY 11210 USA \\ Phone: (718) 951-3857 \\ Fax: (718) 951-4385 \\ e-mail: joshua.fogel@gmail.com \\ * corresponding author
}

